Supplement of Biogeosciences, 17, 317-330, 2020

https://doi.org/10.5194/bg-17-317-2020-supplement

(c) Author(s) 2020. This work is distributed under

the Creative Commons Attribution 4.0 License.

(c) (1)

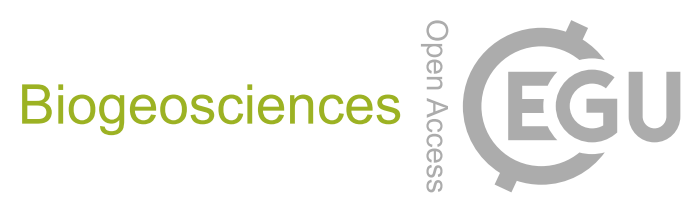

Supplement of

\title{
Structural elucidation and environmental distributions of butanetriol and pentanetriol dialkyl glycerol tetraethers (BDGTs and PDGTs)
}

Sarah Coffinet et al.

Correspondence to: Sarah Coffinet (scoffinet@ marum.de)

The copyright of individual parts of the supplement might differ from the CC BY 4.0 License. 
Supplementary Table S1: Sample general properties, organic matter and geochemical parameters.

\begin{tabular}{|c|c|c|c|c|c|c|c|c|c|c|c|c|c|c|c|}
\hline $\begin{array}{l}\# \text { in } \\
\text { dataset }\end{array}$ & core ID & $\begin{array}{l}\text { latitude } \\
\text { (decimal) }\end{array}$ & $\begin{array}{l}\text { longitude } \\
\text { (decimal) }\end{array}$ & $\begin{array}{l}\text { water } \\
\text { depth }(\mathrm{m})\end{array}$ & $\begin{array}{l}\text { sample depth } \\
\text { (cmbsf) }\end{array}$ & $\begin{array}{l}\text { estimated } \\
\text { age (ka BP) }\end{array}$ & $\begin{array}{l}\mathrm{CH}_{4} \\
(\mu \mathrm{mol} / \mathrm{L})\end{array}$ & $\begin{array}{l}\mathrm{SO}_{4}^{2-} \\
(\mu \mathrm{mol} / \mathrm{L})\end{array}$ & $\begin{array}{l}\mathrm{HS}^{-} \\
(\mu \mathrm{mol} / \mathrm{L})\end{array}$ & $\begin{array}{l}\mathrm{Fe}^{2+} \\
(\mu \mathrm{mol} / \mathrm{L})\end{array}$ & $\begin{array}{l}\text { TOC } \\
\text { (\%) }\end{array}$ & $\begin{array}{l}\mathrm{C} / \mathrm{N} \text { (molar } \\
\text { ratio) }\end{array}$ & $\begin{array}{l}\delta^{13} C_{\text {TOC }} \\
(\%)\end{array}$ & $\begin{array}{l}\text { DOC } \\
(\mu \mathrm{mol} / \mathrm{L})\end{array}$ & $\begin{array}{l}\text { DIC } \\
(\mu \mathrm{mol} / \mathrm{L})\end{array}$ \\
\hline 1 & GeoB15103-1 & 33.03 & 32.63 & 1424 & 3.0 & 0.68 & 0.002 & 30600 & 5.0 & 0.00 & 0.27 & 15.8 & -18.3 & 382 & 2710 \\
\hline 2 & GeoB15103-1 & 33.03 & 32.63 & 1424 & 26.5 & 9.30 & 0.30 & 30800 & 5.0 & 14.0 & 1.58 & 15.4 & -20.9 & 755 & 3140 \\
\hline 3 & GeoB15103-1 & 33.03 & 32.63 & 1424 & 297 & 73.15 & 0.16 & 28600 & 5.0 & 54.3 & 0.16 & 9.30 & -17.8 & 768 & 4030 \\
\hline 4 & GeoB15103-1 & 33.03 & 32.63 & 1424 & 314 & 77.21 & 0.28 & 28500 & 5.0 & 46.5 & 2.33 & 18.1 & -19.6 & 1243 & 4060 \\
\hline 5 & GeoB15103-1 & 33.03 & 32.63 & 1424 & 460 & 121.05 & 0.82 & 27600 & 5.0 & 18.5 & 4.34 & 20.3 & -20.1 & 1047 & 4660 \\
\hline 6 & GeoB15103-2 & 33.03 & 32.63 & 1367 & 27.5 & 9.30 & 0.08 & 29800 & 5.3 & 0.00 & 1.89 & 17.0 & -21.2 & 428 & 2770 \\
\hline 7 & GeoB15103-2 & 33.03 & 32.63 & 1367 & 230 & 60.24 & 0.28 & 28500 & 5.9 & 31.4 & 0.50 & 19.4 & -19.9 & 373 & 3640 \\
\hline 8 & GeoB15103-2 & 33.03 & 32.63 & 1367 & 293 & 77.21 & 0.26 & 28200 & 5.6 & 48.8 & 2.02 & 18.1 & -19.5 & 797 & 3760 \\
\hline 9 & GeoB15103-2 & 33.03 & 32.63 & 1367 & 445 & 121.05 & 0.10 & 27800 & 5.4 & 25.7 & 3.77 & 20.9 & -20.7 & 1388 & 4000 \\
\hline 10 & GeoB15103-2 & 33.03 & 32.63 & 1367 & 473 & 131.90 & 0.54 & 27500 & 5.4 & 25.6 & 0.10 & 11.7 & -20.2 & 5332 & 4060 \\
\hline 11 & GeoB15103-2 & 33.03 & 32.63 & 1367 & 559 & 173.30 & 0.64 & 26500 & 5.4 & 18.9 & 1.31 & 17.0 & -21.2 & 1663 & 4000 \\
\hline 12 & GeoB15104-1 & 40.80 & 27.72 & 606 & 3.0 & 0.05 & 0.17 & 30400 & 5.3 & 0.20 & 0.86 & 10.0 & -22.9 & 438 & 2890 \\
\hline 13 & GeoB15104-1 & 40.80 & 27.72 & 606 & 35 & 0.63 & 0.30 & 30800 & 5.7 & 14.4 & 0.69 & 10.1 & -22.9 & 166 & 3070 \\
\hline 14 & GeoB15104-2 & 40.80 & 27.72 & 600 & 188 & 4.76 & 0.70 & 29500 & 9.1 & 1.00 & 1.03 & 13.4 & -24.1 & 286 & 3620 \\
\hline 15 & GeoB15104-2 & 40.80 & 27.72 & 600 & 288 & 10.10 & 0.80 & 29900 & 5.9 & 0.00 & 0.87 & 14.5 & -25.4 & 322 & 3420 \\
\hline 16 & GeoB15104-2 & 40.80 & 27.72 & 600 & 368 & 14.99 & 0.20 & 29200 & 5.7 & 0.30 & 0.45 & 13.1 & -25.8 & 251 & 4170 \\
\hline 17 & GeoB15104-2 & 40.80 & 27.72 & 600 & 628 & 19.87 & 2.2 & 26700 & 5.6 & 27.6 & 0.58 & 11.3 & -26.1 & 543 & 5490 \\
\hline 18 & GeoB15105-4 & 41.53 & 30.88 & 1266 & 9.0 & 0.23 & 9.6 & 12800 & 1700 & 0.30 & 1.26 & 10.5 & -24.5 & 1053 & 9080 \\
\hline 19 & GeoB15105-4 & 41.53 & 30.88 & 1266 & 39 & 0.92 & 26.5 & 10200 & 3430 & 0.20 & 1.20 & 10.8 & -24.8 & 522 & 12000 \\
\hline 20 & GeoB15105-2 & 41.53 & 30.88 & 1266 & 113 & 2.60 & 1230 & 2810 & 5600 & 0.20 & 1.32 & 11.8 & -24.8 & 2070 & 22000 \\
\hline 21 & GeoB15105-2 & 41.53 & 30.88 & 1266 & 155 & 3.57 & 920 & 2690 & 4580 & 0.20 & 1.43 & 11.9 & -24.7 & 3340 & 21800 \\
\hline 22 & GeoB15105-2 & 41.53 & 30.88 & 1266 & 273 & 5.31 & 1120 & 2040 & 2005 & 0.40 & 1.88 & 12.9 & -24.3 & 2120 & 22000 \\
\hline 23 & GeoB15105-2 & 41.53 & 30.88 & 1266 & 398 & 7.16 & 850 & 1330 & 680 & 2.50 & 4.37 & 15.4 & -24.5 & 4180 & 20800 \\
\hline 24 & GeoB15105-2 & 41.53 & 30.88 & 1266 & 428 & 7.84 & 920 & 965 & 265 & 2.00 & 1.23 & 11.0 & -26.1 & 2990 & 21600 \\
\hline 25 & GeoB15105-2 & 41.53 & 30.88 & 1266 & 605 & 15.13 & 814 & 660 & 120 & 54.0 & 1.12 & 11.9 & -26.1 & 2300 & 18050 \\
\hline 26 & GeoB17302-5 & 42.33 & 3.48 & 741 & 3.5 & 0.04 & 0.17 & 30400 & 0.0 & 60.1 & 0.58 & 9.70 & -22.8 & 265 & 1950 \\
\hline 27 & GeoB17302-5 & 42.33 & 3.48 & 741 & 60 & 0.60 & 0.26 & 28200 & 0.0 & 71.6 & 0.30 & 11.7 & -23.9 & 274 & 1240 \\
\hline 28 & GeoB17302-5 & 42.33 & 3.48 & 741 & 180 & 1.80 & 0.27 & 27000 & 0.0 & 65.4 & 0.30 & 11.7 & -24.0 & 289 & 1260 \\
\hline
\end{tabular}




\begin{tabular}{|c|c|c|c|c|c|c|c|c|c|c|c|c|c|c|c|}
\hline 29 & GeoB17304-3 & 41.99 & 4.84 & 2291 & 8.0 & 1.87 & 0.00 & 30200 & 0.0 & 0.00 & 0.15 & 5.80 & -22.3 & 225 & 838 \\
\hline 30 & GeoB17304-3 & 41.99 & 4.84 & 2291 & 19.5 & 3.36 & 0.00 & 29700 & 0.0 & 9.44 & 0.08 & 4.70 & -22.2 & 181 & 1684 \\
\hline 31 & GeoB17304-3 & 41.99 & 4.84 & 2291 & 57.5 & 7.25 & 0.00 & 29400 & 0.0 & 28.3 & 0.15 & 17.5 & -22.8 & 294 & 2320 \\
\hline 32 & GeoB17304-3 & 41.99 & 4.84 & 2291 & 180 & 17.09 & 0.00 & 21100 & 0.0 & 83.9 & 0.26 & 15.2 & -24.5 & 486 & 1065 \\
\hline 33 & GeoB17306-1 & 43.32 & 4.87 & 30 & 4.5 & 0.00 & 7.56 & 36600 & 0.0 & 45.0 & 1.31 & 13.9 & -26.7 & 351 & 1190 \\
\hline 34 & GeoB17306-1 & 43.32 & 4.87 & 30 & 32.5 & 0.00 & 28.5 & 25200 & 0.0 & 31.9 & 1.42 & 15.1 & -26.9 & 715 & 8240 \\
\hline 35 & GeoB17306-2 & 43.32 & 4.87 & 29 & 70 & 0.00 & 295 & 3260 & 0.0 & 281 & 1.16 & 13.5 & -26.3 & 2252 & 31500 \\
\hline 36 & GeoB17306-2 & 43.32 & 4.87 & 29 & 255 & 0.01 & 3500 & 880 & 0.0 & 224 & 1.40 & 14.8 & -26.9 & 3571 & 22600 \\
\hline 37 & GeoB17306-2 & 43.32 & 4.87 & 29 & 382 & 0.01 & 4440 & 810 & 0.0 & 426 & 0.58 & 33.8 & -24.2 & 3936 & 25100 \\
\hline 38 & GeoB17306-2 & 43.32 & 4.87 & 29 & 430 & 0.01 & 2030 & 396 & 0.0 & 207 & 1.21 & 14.1 & -25.9 & 3413 & 29100 \\
\hline 39 & GeoB17307-5 & 43.30 & 4.86 & 52 & 4.5 & 0.00 & 6.14 & 30500 & 0.0 & 206 & 1.21 & 14.1 & -26.6 & 241 & 1200 \\
\hline 40 & GeoB17307-5 & 43.30 & 4.86 & 52 & 22.5 & 0.00 & 26.0 & 15000 & 0.0 & 78.1 & 1.08 & 14.0 & -26.4 & 659 & 14200 \\
\hline 41 & GeoB17307-8 & 43.30 & 4.86 & 52 & 62.5 & 0.00 & 8.18 & 13700 & 0.0 & 7.69 & 1.00 & 14.6 & -26.3 & 1529 & 16700 \\
\hline 42 & GeoB17307-8 & 43.30 & 4.86 & 52 & 108 & 0.01 & 2150 & 4920 & 0.0 & 39.8 & 0.73 & 12.2 & -26.2 & 3220 & 20600 \\
\hline 43 & GeoB17307-8 & 43.30 & 4.86 & 52 & 339 & 0.02 & 3500 & 0.0 & 0.0 & 269 & 1.05 & 15.3 & -25.9 & 3298 & 27700 \\
\hline 44 & GeoB17307-8 & 43.30 & 4.86 & 52 & 446 & 0.03 & 1520 & 0.0 & 0.0 & 103 & 1.39 & 16.2 & -26.0 & 3119 & 20100 \\
\hline 45 & GeoB17308-1 & 43.27 & 4.73 & 62 & 1.5 & 0.01 & 1.86 & 30800 & 0.0 & 53.4 & 0.91 & 11.8 & -24.9 & 133 & 1780 \\
\hline 46 & GeoB17308-4 & 43.27 & 4.73 & 61 & 25.0 & 0.04 & 1.33 & 27300 & 0.0 & 168 & 0.63 & 12.3 & -24.7 & 422 & 2790 \\
\hline 47 & GeoB17308-4 & 43.27 & 4.73 & 61 & 225 & 0.36 & 60.7 & 3880 & 0.0 & 55.4 & 0.50 & 9.70 & -24.7 & 1078 & 6760 \\
\hline 48 & GeoB17308-4 & 43.27 & 4.73 & 61 & 275 & 0.44 & 72.4 & 1050 & 0.0 & 74.2 & 0.51 & 9.90 & -25.0 & 1334 & 4760 \\
\hline
\end{tabular}


Supplementary Table S2: Absolute concentration, relative abundance and distribution indices of GDGTs, BDGTs and PDGTs. (n.d.: not detected; n.a.: not applicable).

\begin{tabular}{|c|c|c|c|c|c|c|c|c|c|c|c|c|c|c|c|}
\hline $\begin{array}{c}\text { \# in } \\
\text { dataset }\end{array}$ & IGSN & core ID & $\begin{array}{c}\text { sample } \\
\text { depth } \\
\text { (cmbsf) }\end{array}$ & $\begin{array}{c}\text { [2G- } \\
\text { BDGTs] } \\
(\mu \mathrm{g} / \mathrm{g} \\
\mathrm{OC}) \\
\end{array}$ & $\begin{array}{c}\text { [1G- } \\
\text { BDGTs] } \\
(\mu \mathrm{g} / \mathrm{g} \\
\mathrm{OC})\end{array}$ & $\begin{array}{c}\text { [CL- } \\
\text { BDGT-0] } \\
(\mu \mathrm{g} / \mathrm{g} \\
\mathrm{OC}) \\
\end{array}$ & $\begin{array}{c}\text { [CL- } \\
\text { BDGT-1] } \\
(\mu \mathrm{g} / \mathrm{g} \\
\mathrm{OC})\end{array}$ & $\begin{array}{c}\text { [CL- } \\
\text { BDGT-2] } \\
(\mu \mathrm{g} / \mathrm{g} \\
\mathrm{OC})\end{array}$ & $\begin{array}{c}\text { sum }[C L- \\
\text { BDGTs] } \\
(\mu \mathrm{g} / \mathrm{g} \\
\mathrm{OC})\end{array}$ & $\begin{array}{c}\text { sum [IPL- } \\
\text { BDGTs] } \\
(\mu \mathrm{g} / \mathrm{g} \\
\mathrm{OC})\end{array}$ & $\begin{array}{c}\text { sum } \\
\text { [BDGTs] } \\
(\mu \mathrm{g} / \mathrm{g} \\
\mathrm{OC})\end{array}$ & $\begin{array}{c}\text { f(2G- } \\
\text { BDGTs) } \\
(\%)\end{array}$ & $\begin{array}{c}\text { f(1G- } \\
\text { BDGTs) } \\
(\%)\end{array}$ & $\begin{array}{c}\text { f(IPL- } \\
\text { BDGTs }) \\
(\%)\end{array}$ & $\begin{array}{c}f(C L- \\
\text { BDGTs) } \\
(\%)\end{array}$ \\
\hline 1 & IGSN: GEOB0151EXIH101 & GeoB15103-3 & 3 & n.d. & n.d. & n.d. & n.d. & n.d. & n.a. & n.a. & n.a. & n.a. & n.a. & n.a. & n.a. \\
\hline 2 & IGSN: GEOB0151EXCG101 & GeoB15103-1 & 26.5 & 1.55 & 1.15 & 0.99 & 0.35 & 0.10 & 1.45 & 2.71 & 4.15 & 37 & 28 & 65 & 35 \\
\hline 3 & IGSN: GEOB0151EXJG101 & GeoB15103-1 & 297 & n.d. & 0.20 & 0.73 & n.d. & n.d. & 0.73 & 0.20 & 0.93 & n.a. & 22 & 22 & 78 \\
\hline 4 & IGSN: GEOB0151EXKG101 & GeoB15103-1 & 314 & 3.26 & 2.08 & 0.48 & 0.22 & 0.05 & 0.76 & 5.35 & 6.11 & 53 & 34 & 88 & 12 \\
\hline 5 & IGSN: GEOB0151EXQG101 & GeoB15103-1 & 460 & 2.02 & 2.40 & 1.59 & 0.48 & 0.16 & 2.23 & 4.42 & 6.65 & 30 & 36 & 66 & 34 \\
\hline 6 & IGSN: GEOB0151EХOH101 & GeoB15103-2 & 27.5 & 0.97 & 1.18 & 0.16 & 0.07 & n.d. & 0.23 & 2.15 & 2.37 & 41 & 50 & 90 & 10 \\
\hline 7 & IGSN: GEOB0151EX5H101 & GeoB15103-2 & 230 & n.d. & 0.02 & 0.005 & n.d. & 0.03 & 0.03 & 0.02 & 0.05 & n.a. & 39 & 39 & 61 \\
\hline 8 & IGSN: GEOB0151EX7H101 & GeoB15103-2 & 293 & 2.15 & 3.20 & 0.28 & 0.09 & 0.03 & 0.39 & 5.35 & 5.74 & 37 & 56 & 93 & 7 \\
\hline 9 & IGSN: GEOB0151EXBH101 & GeoB15103-2 & 445 & 0.98 & 2.02 & 0.33 & 0.16 & 0.04 & 0.52 & 3.00 & 3.53 & 28 & 57 & 85 & 15 \\
\hline 10 & IGSN: GEOB0151EXCH101 & GeoB15103-2 & 473 & 0.13 & 0.24 & 0.05 & n.d. & 0.05 & 0.10 & 0.36 & 0.46 & 27 & 51 & 78 & 22 \\
\hline 11 & IGSN: GEOB0151EXEH101 & GeoB15103-2 & 559 & n.d. & 0.81 & 0.27 & 0.02 & 0.02 & 0.31 & 0.81 & 1.12 & n.a. & 72 & 72 & 28 \\
\hline 12 & IGSN: GEOB0151EX5J101 & GeoB15104-1 & 3.0 & 0.09 & 0.45 & 0.25 & 0.06 & 0.02 & 0.32 & 0.53 & 0.86 & 10 & 52 & 62 & 38 \\
\hline 13 & IGSN: GEOB0151EXப101 & GeoB15104-1 & 35 & 0.28 & 0.53 & 0.24 & 0.03 & n.d. & 0.26 & 0.81 & 1.08 & 26 & 49 & 76 & 24 \\
\hline 14 & IGSN: GEOB0151EXVJ101 & GeoB15104-2 & 188 & 0.27 & 0.41 & 0.30 & 0.12 & 0.01 & 0.43 & 0.68 & 1.11 & 24 & 37 & 61 & 39 \\
\hline 15 & IGSN: GEOB0151EXXJ101 & GeoB15104-2 & 288 & 1.34 & 1.59 & 0.33 & 0.17 & n.d. & 0.50 & 2.93 & 3.43 & 39 & 46 & 85 & 15 \\
\hline 16 & IGSN: GEOB0151EХ0K101 & GeoB15104-2 & 368 & 0.24 & 0.20 & 0.80 & 0.10 & n.d. & 0.90 & 0.44 & 1.34 & 18 & 15 & 33 & 67 \\
\hline 17 & IGSN: GEOB0151EX5K101 & GeoB15104-2 & 628 & n.d. & n.d. & 0.35 & 0.01 & n.d. & 0.36 & n.a. & 0.36 & n.a. & n.a. & n.a. & 100 \\
\hline 18 & IGSN: GEOB0151EXAM101 & GeoB15105-4 & 9.0 & 0.77 & 5.69 & 1.92 & 0.51 & 0.25 & 2.69 & 6.46 & 9.15 & 8 & 62 & 71 & 29 \\
\hline 19 & IGSN: GEOB0151EXPM101 & GeoB15105-4 & 39 & 0.32 & 1.75 & 2.03 & 0.60 & 0.39 & 3.02 & 2.07 & 5.09 & 6 & 34 & 41 & 59 \\
\hline 20 & IGSN: GEOB0151EXOL101 & GeoB15105-2 & 113 & 0.81 & 2.18 & 1.70 & 0.46 & 0.15 & 2.32 & 2.99 & 5.31 & 15 & 41 & 56 & 44 \\
\hline 21 & IGSN: GEOB0151EX1L101 & GeoB15105-2 & 155 & 0.54 & 1.72 & 1.50 & 0.49 & 0.18 & 2.17 & 2.26 & 4.43 & 12 & 39 & 51 & 49 \\
\hline 22 & IGSN: GEOB0151EX4L101 & GeoB15105-2 & 273 & 0.25 & 1.44 & 1.20 & 0.44 & 0.12 & 1.76 & 1.69 & 3.45 & 7 & 42 & 49 & 51 \\
\hline 23 & IGSN: GEOB0151EX6L101 & GeoB15105-2 & 398 & n.d. & 1.17 & 0.46 & 0.15 & 0.04 & 0.65 & 1.17 & 1.82 & n.a. & 64 & 64 & 36 \\
\hline 24 & IGSN: GEOB0151EX8L101 & GeoB15105-2 & 428 & n.d. & 1.31 & 0.36 & 0.22 & 0.08 & 0.65 & 1.31 & 1.95 & n.a. & 67 & 67 & 33 \\
\hline 25 & IGSN: GEOB0151EXCL101 & GeoB15105-2 & 605 & n.d. & 0.16 & 0.10 & 0.04 & 0.03 & 0.17 & 0.16 & 0.33 & n.a. & 48 & 48 & 52 \\
\hline 26 & IGSN: GEOB0173EXYS001 & GeoB17302-5 & 3.5 & 1.17 & 0.98 & 0.11 & 0.03 & 0.01 & 0.15 & 2.15 & 2.30 & 51 & 42 & 93 & 7 \\
\hline 27 & IGSN: GEOB0173ЕХ0T001 & GeoB17302-5 & 60 & 0.38 & 0.63 & 0.71 & 0.08 & 0.04 & 0.83 & 1.01 & 1.84 & 21 & 34 & 55 & 45 \\
\hline
\end{tabular}




\begin{tabular}{|c|c|c|c|c|c|c|c|c|c|c|c|c|c|c|c|}
\hline 28 & IGSN: GEOB0173EX4T001 & GeoB17302-5 & 180 & 0.86 & 0.86 & 0.86 & 0.17 & 0.13 & 1.15 & 1.72 & 2.87 & 30 & 30 & 60 & 40 \\
\hline 29 & IGSN: GEOB0173EX2V001 & GeoB17304-3 & 8.0 & n.d. & n.d. & n.d. & n.d. & n.d. & n.a. & n.a. & n.a. & n.a. & n.a. & n.a. & n.a. \\
\hline 30 & IGSN: GEOB0173EX3V001 & GeoB17304-3 & 19.5 & n.d. & n.d. & n.d. & n.d. & n.d. & n.a. & n.a. & n.a. & n.a. & n.a. & n.a. & n.a. \\
\hline 31 & IGSN: GEOB0173EX7V001 & GeoB17304-3 & 57.5 & 0.14 & 0.32 & 0.12 & 0.08 & n.d. & 0.24 & 0.46 & 0.70 & 20 & 45 & 65 & 35 \\
\hline 32 & IGSN: GEOB0173EXMVO01 & GeoB17304-3 & 180 & 0.32 & 0.67 & 0.22 & 0.13 & 0.09 & 0.44 & 0.99 & 1.44 & 22 & 47 & 69 & 31 \\
\hline 33 & IGSN: GEOB0173EXDL001 & GeoB17306-1 & 4.5 & 0.66 & 0.38 & 0.10 & 0.04 & 0.03 & 0.18 & 1.04 & 1.22 & 54 & 31 & 86 & 14 \\
\hline 34 & IGSN: GEOB0173EXKL001 & GeoB17306-1 & 32.5 & 2.50 & 0.79 & 0.12 & 0.04 & 0.03 & 0.19 & 3.28 & 3.47 & 72 & 23 & 95 & 5 \\
\hline 35 & IGSN: GEOB0173EX8E001 & GeoB17306-2 & 70 & 2.89 & 0.77 & 0.13 & 0.04 & 0.02 & 0.19 & 3.66 & 3.85 & 75 & 20 & 95 & 5 \\
\hline 36 & IGSN: GEOB0173EXCE001 & GeoB17306-2 & 255 & 3.33 & 1.53 & 0.54 & 0.10 & 0.03 & 0.67 & 4.86 & 5.53 & 60 & 28 & 88 & 12 \\
\hline 37 & IGSN: GEOB0173EXHE001 & GeoB17306-2 & 382 & 1.21 & 0.32 & 0.14 & 0.07 & 0.05 & 0.25 & 1.54 & 1.79 & 68 & 18 & 86 & 14 \\
\hline 38 & IGSN: GEOB0173EXIE001 & GeoB17306-2 & 430 & 3.29 & 0.82 & 0.12 & 0.03 & 0.01 & 0.16 & 4.12 & 4.28 & 77 & 19 & 96 & 4 \\
\hline 39 & IGSN: GEOB0173EX5M001 & GeoB17307-5 & 4.5 & 1.77 & 0.95 & 0.61 & 0.16 & 0.07 & 0.83 & 2.72 & 3.55 & 50 & 27 & 77 & 23 \\
\hline 40 & IGSN: GEOB0173EXAM001 & GeoB17307-5 & 22.5 & 3.30 & 1.00 & 0.16 & 0.07 & 0.04 & 0.27 & 4.29 & 4.56 & 72 & 22 & 94 & 6 \\
\hline 41 & IGSN: GEOB0173EX8Y001 & GeoB17307-8 & 62.5 & 2.27 & 0.99 & 0.22 & 0.16 & 0.11 & 0.48 & 3.25 & 3.74 & 61 & 26 & 87 & 13 \\
\hline 42 & IGSN: GEOB0173EXBY001 & GeoB17307-8 & 108 & 2.97 & 1.06 & 0.21 & 0.06 & 0.03 & 0.30 & 4.04 & 4.33 & 69 & 25 & 93 & 7 \\
\hline 43 & IGSN: GEOB0173EXMY001 & GeoB17307-8 & 339 & 3.00 & 0.96 & 0.34 & 0.12 & 0.05 & 0.51 & 3.96 & 4.47 & 67 & 21 & 89 & 11 \\
\hline 44 & IGSN: GEOB0173EXPY001 & GeoB17307-8 & 446 & 2.72 & 0.79 & 0.19 & 0.11 & 0.06 & 0.36 & 3.51 & 3.88 & 70 & 20 & 91 & 9 \\
\hline 45 & IGSN: GEOB0173EXCM001 & GeoB17308-1 & 1.5 & 5.01 & 2.21 & 0.70 & 0.15 & 0.08 & 0.93 & 7.22 & 8.15 & 61 & 27 & 89 & 11 \\
\hline 46 & IGSN: GEOB0173EXLZ001 & GeoB17308-4 & 25.0 & 3.22 & 1.48 & 0.26 & 0.06 & 0.03 & 0.34 & 4.70 & 5.04 & 64 & 29 & 93 & 7 \\
\hline 47 & IGSN: GEOB0173EXUZ001 & GeoB17308-4 & 225 & 2.01 & 0.95 & 0.38 & 0.05 & 0.03 & 0.46 & 2.96 & 3.42 & 59 & 28 & 86 & 14 \\
\hline 48 & IGSN: GEOB0173EXWZ001 & GeoB17308-4 & 275 & 1.13 & 0.60 & 0.10 & 0.03 & 0.01 & 0.15 & 1.73 & 1.88 & 60 & 32 & 92 & 8 \\
\hline
\end{tabular}

Supplementary Table S2 (continued): Absolute concentration, relative abundance and distribution indices of GDGTs, BDGTs and PDGTs. (n.d.:

not detected; n.a.: not applicable).

\begin{tabular}{|c|c|c|c|c|c|c|c|c|c|c|c|c|}
\hline $\begin{array}{c}\text { \# in } \\
\text { dataset }\end{array}$ & IGSN & core ID & $\begin{array}{c}\text { sample } \\
\text { depth } \\
\text { (cmbsf) }\end{array}$ & $\begin{array}{c}\text { [2G-PDGTs] } \\
(\mu \mathrm{g} / \mathrm{g} O C)\end{array}$ & $\begin{array}{c}\text { [1G-PDGTs] } \\
(\mu \mathrm{g} / \mathrm{g} \text { OC) }\end{array}$ & $\begin{array}{c}\text { [CL-PDGTs] } \\
(\mu \mathrm{g} / \mathrm{g} \text { OC) }\end{array}$ & $\begin{array}{c}\text { sum [IPL- } \\
\text { PDGTs] } \\
(\mu \mathrm{g} / \mathrm{g} \mathrm{OC})\end{array}$ & $\begin{array}{c}\text { sum } \\
{[\text { PDGTs] }} \\
(\mu \mathrm{g} / \mathrm{g} \mathrm{OC})\end{array}$ & $\begin{array}{c}f(2 G- \\
\text { PDGT) (\%) }\end{array}$ & $\begin{array}{c}f(1 G- \\
\text { PDGT) (\%) }\end{array}$ & $\begin{array}{c}f(C L- \\
\text { PDGT) (\%) }\end{array}$ & $\begin{array}{c}f(I P L- \\
\text { PDGTs) (\%) }\end{array}$ \\
\hline 1 & IGSN: GEOB0151EXIH101 & GeoB15103-3 & 3 & n.d. & 0.87 & n.d. & 0.87 & 0.87 & n.a. & 100 & n.a. & 100 \\
\hline 2 & IGSN: GEOB0151EXCG101 & GeoB15103-1 & 26.5 & 0.20 & 0.55 & 0.19 & 0.76 & 0.95 & 22 & 58 & 20 & 80 \\
\hline 3 & IGSN: GEOB0151EXJG101 & GeoB15103-1 & 297 & n.d. & 0.20 & n.d. & 0.20 & 0.20 & n.a. & 100 & n.a. & 100 \\
\hline 4 & IGSN: GEOB0151EXKG101 & GeoB15103-1 & 314 & 1.54 & 0.40 & 0.13 & 1.94 & 2.07 & 74 & 19 & 6 & 94 \\
\hline 5 & IGSN: GEOB0151EXQG101 & GeoB15103-1 & 460 & 1.09 & 0.96 & 1.05 & 2.05 & 3.10 & 35 & 31 & 34 & 66 \\
\hline 6 & IGSN: GEOB0151EХOH101 & GeoB15103-2 & 27.5 & 0.09 & 0.72 & 0.01 & 0.81 & 0.82 & 10 & 88 & 2 & 98 \\
\hline
\end{tabular}




\begin{tabular}{|c|c|c|c|c|c|c|c|c|c|c|c|c|}
\hline 7 & IGSN: GEOB0151EX5H101 & GeoB15103-2 & 230 & n.d. & n.d. & n.d. & n.a. & n.a. & n.a. & n.a. & n.a. & n.a. \\
\hline 8 & IGSN: GEOB0151EX7H101 & GeoB15103-2 & 293 & 0.90 & 0.68 & n.d. & 1.58 & 1.58 & 57 & 43 & n.a. & 100 \\
\hline 9 & IGSN: GEOB0151EXBH101 & GeoB15103-2 & 445 & 0.43 & 0.65 & 0.17 & 1.08 & 1.25 & 34 & 52 & 14 & 86 \\
\hline 10 & IGSN: GEOB0151EXCH101 & GeoB15103-2 & 473 & 0.22 & 0.24 & n.d. & 0.46 & 0.46 & 49 & 51 & n.a. & 100 \\
\hline 11 & IGSN: GEOB0151EXEH101 & GeoB15103-2 & 559 & 0.43 & 0.22 & 0.10 & 0.65 & 0.75 & 57 & 29 & 13 & 87 \\
\hline 12 & IGSN: GEOB0151EX5J101 & GeoB15104-1 & 3.0 & n.d. & 0.89 & n.d. & 0.89 & 0.89 & n.a. & 100 & n.a. & 100 \\
\hline 13 & IGSN: GEOB0151EXU101 & GeoB15104-1 & 35 & n.d. & 0.91 & 0.02 & 0.91 & 0.93 & n.a. & 98 & 2 & 98 \\
\hline 14 & IGSN: GEOB0151EXVJ101 & GeoB15104-2 & 188 & n.d. & 0.73 & 0.07 & 0.73 & 0.79 & n.a. & 92 & 8 & 92 \\
\hline 15 & IGSN: GEOB0151EXXJ101 & GeoB15104-2 & 288 & n.d. & 0.90 & 0.06 & 0.90 & 0.96 & n.a. & 94 & 6 & 94 \\
\hline 16 & IGSN: GEOB0151EX0K101 & GeoB15104-2 & 368 & n.d. & 0.10 & 0.13 & 0.10 & 0.23 & n.a. & 44 & 56 & 44 \\
\hline 17 & IGSN: GEOB0151EX5K101 & GeoB15104-2 & 628 & n.d. & n.d. & 0.04 & n.a. & 0.04 & n.a. & n.a. & 100 & n.a. \\
\hline 18 & IGSN: GEOB0151EXAM101 & GeoB15105-4 & 9.0 & n.d. & n.d. & n.d. & n.a. & n.a. & n.a. & n.a. & n.a. & n.a. \\
\hline 19 & IGSN: GEOB0151EXPM101 & GeoB15105-4 & 39 & n.d. & n.d. & n.d. & n.a. & n.a. & n.a. & n.a. & n.a. & n.a. \\
\hline 20 & IGSN: GEOB0151EXOL101 & GeoB15105-2 & 113 & n.d. & n.d. & n.d. & n.a. & n.a. & n.a. & n.a. & n.a. & n.a. \\
\hline 21 & IGSN: GEOB0151EX1L101 & GeoB15105-2 & 155 & n.d. & n.d. & n.d. & n.a. & n.a. & n.a. & n.a. & n.a. & n.a. \\
\hline 22 & IGSN: GEOB0151EX4L101 & GeoB15105-2 & 273 & n.d. & n.d. & n.d. & n.a. & n.a. & n.a. & n.a. & n.a. & n.a. \\
\hline 23 & IGSN: GEOB0151EX6L101 & GeoB15105-2 & 398 & n.d. & n.d. & n.d. & n.a. & n.a. & n.a. & n.a. & n.a. & n.a. \\
\hline 24 & IGSN: GEOB0151EX8L101 & GeoB15105-2 & 428 & n.d. & n.d. & n.d. & n.a. & n.a. & n.a. & n.a. & n.a. & n.a. \\
\hline 25 & IGSN: GEOB0151EXCL101 & GeoB15105-2 & 605 & n.d. & n.d. & n.d. & n.a. & n.a. & n.a. & n.a. & n.a. & n.a. \\
\hline 26 & IGSN: GEOB0173EXYS001 & GeoB17302-5 & 3.5 & n.d. & 0.49 & n.d. & 0.49 & 0.49 & n.a. & 100 & n.a. & 100 \\
\hline 27 & IGSN: GEOB0173EX0T001 & GeoB17302-5 & 60 & n.d. & n.d. & 0.20 & n.a. & 0.20 & n.a. & n.a. & 100 & n.a. \\
\hline 28 & IGSN: GEOB0173EX4T001 & GeoB17302-5 & 180 & n.d. & n.d. & 0.40 & n.a. & 0.40 & n.a. & n.a. & 100 & n.a. \\
\hline 29 & IGSN: GEOB0173EX2V001 & GeoB17304-3 & 8.0 & n.d. & 0.35 & n.d. & 0.35 & 0.35 & n.a. & 100 & n.a. & 100 \\
\hline 30 & IGSN: GEOB0173EX3V001 & GeoB17304-3 & 19.5 & n.d. & 0.46 & n.d. & 0.46 & 0.46 & n.a. & 100 & n.a. & 100 \\
\hline 31 & IGSN: GEOB0173EX7V001 & GeoB17304-3 & 57.5 & n.d. & 0.08 & 0.12 & 0.08 & 0.20 & n.a. & 39 & 61 & 39 \\
\hline 32 & IGSN: GEOB0173EXMV001 & GeoB17304-3 & 180 & n.d. & n.d. & 0.10 & n.a. & 0.10 & n.a. & n.a. & 100 & n.a. \\
\hline 33 & IGSN: GEOB0173EXDL001 & GeoB17306-1 & 4.5 & n.d. & n.d. & n.d. & n.a. & n.a. & n.a. & n.a. & n.a. & n.a. \\
\hline 34 & IGSN: GEOB0173EXKL001 & GeoB17306-1 & 32.5 & 0.85 & n.d. & n.d. & 0.85 & 0.85 & 100 & n.a. & n.a. & 100 \\
\hline 35 & IGSN: GEOB0173EX8E001 & GeoB17306-2 & 70 & 0.84 & n.d. & n.d. & 0.84 & 0.84 & 100 & n.a. & n.a. & 100 \\
\hline 36 & IGSN: GEOB0173EXCE001 & GeoB17306-2 & 255 & n.d. & n.d. & n.d. & n.a. & n.a. & n.a. & n.a. & n.a. & n.a. \\
\hline 37 & IGSN: GEOB0173EXHE001 & GeoB17306-2 & 382 & 0.28 & n.d. & n.d. & 0.28 & 0.28 & 100 & n.a. & n.a. & 100 \\
\hline 38 & IGSN: GEOB0173EXIE001 & GeoB17306-2 & 430 & 0.60 & n.d. & n.d. & 0.60 & 0.60 & 100 & n.a. & n.a. & 100 \\
\hline
\end{tabular}




\begin{tabular}{|c|c|c|c|c|c|c|c|c|c|c|c|c|}
\hline 39 & IGSN: GEOB0173EX5M001 & GeoB17307-5 & 4.5 & 0.55 & n.d. & n.d. & 0.55 & 0.55 & 100 & n.a. & n.a. & 100 \\
\hline 40 & IGSN: GEOB0173EXAM001 & GeoB17307-5 & 22.5 & 0.62 & n.d. & n.d. & 0.62 & 0.62 & 100 & n.a. & n.a. & 100 \\
\hline 41 & IGSN: GEOB0173EX8Y001 & GeoB17307-8 & 62.5 & 1.02 & n.d. & n.d. & 1.02 & 1.02 & 100 & n.a. & n.a. & 100 \\
\hline 42 & IGSN: GEOB0173EXBY001 & GeoB17307-8 & 108 & 1.55 & n.d. & n.d. & 1.55 & 1.55 & 100 & n.a. & n.a. & 100 \\
\hline 43 & IGSN: GEOB0173EXMY001 & GeoB17307-8 & 339 & 1.09 & n.d. & n.d. & 1.09 & 1.09 & 100 & n.a. & n.a. & 100 \\
\hline 44 & IGSN: GEOB0173EXPY001 & GeoB17307-8 & 446 & 0.92 & n.d. & n.d. & 0.92 & 0.92 & 100 & n.a. & n.a. & 100 \\
\hline 45 & IGSN: GEOB0173EXCM001 & GeoB17308-1 & 1.5 & 1.23 & n.d. & n.d. & 1.23 & 1.23 & 100 & n.a. & n.a. & 100 \\
\hline 46 & IGSN: GEOB0173EXLZ001 & GeoB17308-4 & 25.0 & 1.23 & n.d. & n.d. & 1.23 & 1.23 & 100 & n.a. & n.a. & 100 \\
\hline 47 & IGSN: GEOB0173EXUZ001 & GeoB17308-4 & 225 & 0.72 & n.d. & n.d. & 0.72 & 0.72 & 100 & n.a. & n.a. & 100 \\
\hline 48 & IGSN: GEOB0173EXWZ001 & GeoB17308-4 & 275 & 0.57 & n.d. & n.d. & 0.57 & 0.57 & 100 & n.a. & n.a. & 100 \\
\hline
\end{tabular}

Supplementary Table S2 (continued): Absolute concentration, relative abundance and distribution indices of GDGTs, BDGTs and PDGTs. (n.d.: not detected; n.a.: not applicable).

\begin{tabular}{|c|c|c|c|c|c|c|c|c|c|c|c|c|}
\hline $\begin{array}{c}\text { \# in } \\
\text { dataset }\end{array}$ & IGSN & core ID & $\begin{array}{l}\text { sample } \\
\text { depth } \\
\text { (cmbsf) }\end{array}$ & $\begin{array}{l}\text { [2G-GDGT- } \\
0](\mu \mathrm{g} / \mathrm{g} O C)\end{array}$ & $\begin{array}{c}\text { [1G-GDGT-0] } \\
(\mu \mathrm{g} g-1 \mathrm{OC})\end{array}$ & $\begin{array}{c}\text { [CL-GDGT-0] } \\
(\mu \mathrm{g} g-1 \mathrm{OC})\end{array}$ & $\begin{array}{c}\text { sum [IPL- } \\
\text { GDGT-0] } \\
(\mu \mathrm{g} / \mathrm{g} O C) \\
\end{array}$ & $\begin{array}{c}\text { sum } \\
{[\mathrm{GDGT}-0]} \\
(\mu \mathrm{g} / \mathrm{g} \mathrm{OC}) \\
\end{array}$ & $\begin{array}{c}\text { f(GDGT- } \\
0)(\%)\end{array}$ & $\begin{array}{c}f(I P L- \\
\text { GDGT-0) } \\
(\%) \\
\end{array}$ & $\begin{array}{c}\text { (sum- } \\
\text { BDGTs)/(sum- } \\
\text { GDGT-0) (\%) }\end{array}$ & $\begin{array}{c}\text { (sum- } \\
\text { PDGTs)/(sum- } \\
\text { GDGT-0) (\%) }\end{array}$ \\
\hline 1 & IGSN: GEOB0151EXIH101 & GeoB15103-3 & 3 & n.d. & 0.48 & 4.42 & 0.48 & 4.91 & 90.14 & 9.86 & n.a. & 17.7 \\
\hline 2 & IGSN: GEOB0151EXCG101 & GeoB15103-1 & 26.5 & 2.63 & 6.14 & 358.44 & 8.77 & 367.21 & 97.61 & 2.39 & 1.1 & 0.3 \\
\hline 3 & IGSN: GEOB0151EXJG101 & GeoB15103-1 & 297 & n.d. & 1.35 & 5.94 & 1.35 & 7.29 & 81.43 & 18.57 & 12.8 & 2.7 \\
\hline 4 & IGSN: GEOB0151EXKG101 & GeoB15103-1 & 314 & 5.96 & 7.57 & 341.97 & 13.52 & 355.49 & 96.20 & 3.80 & 1.7 & 0.6 \\
\hline 5 & IGSN: GEOB0151EXQG101 & GeoB15103-1 & 460 & 5.76 & 9.29 & 315.86 & 15.04 & 330.91 & 95.45 & 4.55 & 2.0 & 0.9 \\
\hline 6 & IGSN: GEOB0151EXOH101 & GeoB15103-2 & 27.5 & 1.38 & 8.09 & 65.05 & 9.47 & 74.52 & 87.29 & 12.71 & 3.2 & 1.1 \\
\hline 7 & IGSN: GEOB0151EX5H101 & GeoB15103-2 & 230 & n.d. & 0.53 & 1.83 & 0.53 & 2.36 & 77.68 & 22.32 & 1.6 & n.a. \\
\hline 8 & IGSN: GEOB0151EX7H101 & GeoB15103-2 & 293 & 2.58 & 8.81 & 150.82 & 11.40 & 162.22 & 92.98 & 7.02 & 3.5 & 1.0 \\
\hline 9 & IGSN: GEOB0151EXBH101 & GeoB15103-2 & 445 & 1.96 & 8.94 & 163.56 & 10.91 & 174.47 & 93.75 & 6.25 & 2.0 & 0.7 \\
\hline 10 & IGSN: GEOB0151EXCH101 & GeoB15103-2 & 473 & n.d. & 4.27 & 11.01 & 4.27 & 15.28 & 72.06 & 27.94 & 3.0 & 3.0 \\
\hline 11 & IGSN: GEOB0151EXEH101 & GeoB15103-2 & 559 & 0.52 & 7.26 & 95.22 & 7.79 & 103.00 & 92.44 & 7.56 & 1.1 & 0.7 \\
\hline 12 & IGSN: GEOB0151EX5J101 & GeoB15104-1 & 3.0 & 0.12 & 1.12 & 48.38 & 1.25 & 49.62 & 97.49 & 2.51 & 1.7 & 1.8 \\
\hline 13 & IGSN: GEOB0151EXப101 & GeoB15104-1 & 35 & 0.51 & 3.87 & 74.68 & 4.38 & 79.06 & 94.46 & 5.54 & 1.4 & 1.2 \\
\hline 14 & IGSN: GEOB0151EXVJ101 & GeoB15104-2 & 188 & 0.28 & 3.75 & 52.87 & 4.02 & 56.89 & 92.93 & 7.07 & 1.9 & 1.4 \\
\hline 15 & IGSN: GEOB0151EXXJ101 & GeoB15104-2 & 288 & 4.08 & 13.75 & 147.76 & 17.83 & 165.59 & 89.23 & 10.77 & 2.1 & 0.6 \\
\hline 16 & IGSN: GEOB0151EХОК101 & GeoB15104-2 & 368 & 0.08 & 1.14 & 47.59 & 1.22 & 48.81 & 97.51 & 2.49 & 2.7 & 0.5 \\
\hline 17 & IGSN: GEOB0151EX5K101 & GeoB15104-2 & 628 & n.d. & 3.13 & 18.80 & 3.13 & 21.93 & 85.73 & 14.27 & 1.7 & 0.2 \\
\hline
\end{tabular}




\begin{tabular}{|c|c|c|c|c|c|c|c|c|c|c|c|c|}
\hline 18 & IGSN: GEOB0151EXAM101 & GeoB15105-4 & 9.0 & 5.40 & 25.24 & 364.58 & 30.65 & 395.23 & 92.25 & 7.75 & 2.3 & n.a. \\
\hline 19 & IGSN: GEOB0151EXPM101 & GeoB15105-4 & 39 & 7.98 & 23.26 & 262.00 & 31.24 & 293.24 & 89.35 & 10.65 & 1.7 & n.a. \\
\hline 20 & IGSN: GEOB0151EX0L101 & GeoB15105-2 & 113 & 6.36 & 29.87 & 360.43 & 36.24 & 396.67 & 90.86 & 9.14 & 1.3 & n.a. \\
\hline 21 & IGSN: GEOB0151EX1L101 & GeoB15105-2 & 155 & 6.23 & 22.59 & 287.18 & 28.82 & 316.00 & 90.88 & 9.12 & 1.4 & n.a. \\
\hline 22 & IGSN: GEOB0151EX4L101 & GeoB15105-2 & 273 & 2.93 & 23.27 & 264.75 & 26.21 & 290.96 & 90.99 & 9.01 & 1.2 & n.a. \\
\hline 23 & IGSN: GEOB0151EX6L101 & GeoB15105-2 & 398 & 1.68 & 15.47 & 372.46 & 17.16 & 389.62 & 95.60 & 4.40 & 0.5 & n.a. \\
\hline 24 & IGSN: GEOB0151EX8L101 & GeoB15105-2 & 428 & 0.21 & 3.65 & 173.44 & 3.86 & 177.30 & 97.82 & 2.18 & 1.1 & n.a. \\
\hline 25 & IGSN: GEOB0151EXCL101 & GeoB15105-2 & 605 & 0.36 & 4.02 & 121.51 & 4.38 & 125.89 & 96.52 & 3.48 & 0.3 & n.a. \\
\hline 26 & IGSN: GEOB0173EXYS001 & GeoB17302-5 & 3.5 & 1.45 & 4.29 & 20.27 & 5.74 & 26.01 & 77.94 & 22.06 & 8.9 & 1.9 \\
\hline 27 & IGSN: GEOB0173EX0T001 & GeoB17302-5 & 60 & 0.42 & 2.18 & 122.19 & 2.60 & 124.79 & 97.92 & 2.08 & 1.5 & 0.2 \\
\hline 28 & IGSN: GEOB0173EX4T001 & GeoB17302-5 & 180 & 0.72 & 2.93 & 157.17 & 3.65 & 160.81 & 97.73 & 2.27 & 1.8 & 0.2 \\
\hline 29 & IGSN: GEOB0173EX2V001 & GeoB17304-3 & 8.0 & n.d. & 0.22 & 3.37 & 0.22 & 3.59 & 93.93 & 6.07 & n.a. & 9.8 \\
\hline 30 & IGSN: GEOB0173EX3V001 & GeoB17304-3 & 19.5 & n.d. & 0.24 & 4.24 & 0.24 & 4.48 & 94.71 & 5.29 & n.a. & 10.4 \\
\hline 31 & IGSN: GEOB0173EX7V001 & GeoB17304-3 & 57.5 & 0.06 & 0.99 & 28.64 & 1.05 & 29.69 & 96.47 & 3.53 & 2.4 & 0.7 \\
\hline 32 & IGSN: GEOB0173EXMV001 & GeoB17304-3 & 180 & 0.26 & 1.21 & 17.27 & 1.47 & 18.74 & 92.16 & 7.84 & 7.7 & 0.5 \\
\hline 33 & IGSN: GEOB0173EXDL001 & GeoB17306-1 & 4.5 & 2.89 & 4.53 & 16.61 & 7.42 & 24.03 & 69.12 & 30.88 & 5.1 & n.a. \\
\hline 34 & IGSN: GEOB0173EXKL001 & GeoB17306-1 & 32.5 & 3.11 & 4.82 & 10.10 & 7.93 & 18.03 & 56.02 & 43.98 & 19.2 & 4.7 \\
\hline 35 & IGSN: GEOB0173EX8E001 & GeoB17306-2 & 70 & 2.61 & 4.09 & 8.74 & 6.70 & 15.44 & 56.61 & 43.39 & 24.9 & 5.4 \\
\hline 36 & IGSN: GEOB0173EXCE001 & GeoB17306-2 & 255 & 5.65 & 9.41 & 14.15 & 15.06 & 29.22 & 48.44 & 51.56 & 18.9 & n.a. \\
\hline 37 & IGSN: GEOB0173EXHE001 & GeoB17306-2 & 382 & 0.85 & 1.01 & 26.66 & 1.85 & 28.52 & 93.50 & 6.50 & 6.3 & 1.0 \\
\hline 38 & IGSN: GEOB0173EXIE001 & GeoB17306-2 & 430 & 6.76 & 7.69 & 0.92 & 14.46 & 15.37 & 5.96 & 94.04 & 27.9 & 3.9 \\
\hline 39 & IGSN: GEOB0173EX5M001 & GeoB17307-5 & 4.5 & 5.50 & 10.02 & 20.92 & 15.52 & 36.44 & 57.41 & 42.59 & 9.7 & 1.5 \\
\hline 40 & IGSN: GEOB0173EXAM001 & GeoB17307-5 & 22.5 & 2.38 & 5.49 & 22.42 & 7.87 & 30.29 & 74.02 & 25.98 & 15.1 & 2.0 \\
\hline 41 & IGSN: GEOB0173EX8Y001 & GeoB17307-8 & 62.5 & 2.64 & 3.71 & 9.42 & 6.35 & 15.77 & 59.74 & 40.26 & 23.7 & 6.5 \\
\hline 42 & IGSN: GEOB0173EXBY001 & GeoB17307-8 & 108 & 3.26 & 4.45 & 8.13 & 7.71 & 15.84 & 51.33 & 48.67 & 27.3 & 9.8 \\
\hline 43 & IGSN: GEOB0173EXMY001 & GeoB17307-8 & 339 & 4.41 & 5.56 & 7.66 & 9.97 & 17.63 & 43.45 & 56.55 & 25.4 & 6.2 \\
\hline 44 & IGSN: GEOB0173EXPY001 & GeoB17307-8 & 446 & 2.74 & 3.50 & 9.03 & 6.24 & 15.26 & 59.13 & 40.87 & 25.4 & 6.0 \\
\hline 45 & IGSN: GEOB0173EXCM001 & GeoB17308-1 & 1.5 & 2.39 & 14.36 & 120.32 & 16.75 & 137.07 & 87.78 & 12.22 & 5.9 & 0.9 \\
\hline 46 & IGSN: GEOB0173EXLZ001 & GeoB17308-4 & 25.0 & 1.34 & 7.11 & 29.09 & 8.45 & 37.54 & 77.50 & 22.50 & 13.4 & 3.3 \\
\hline 47 & IGSN: GEOB0173EXUZ001 & GeoB17308-4 & 225 & 0.50 & 2.37 & 20.72 & 2.87 & 23.59 & 87.84 & 12.16 & 14.5 & 3.1 \\
\hline 48 & IGSN: GEOB0173EXWZ001 & GeoB17308-4 & 275 & 0.49 & 2.38 & 10.23 & 2.87 & 13.11 & 78.09 & 21.91 & 14.3 & 4.3 \\
\hline
\end{tabular}


Supplementary Table S3: Carbon isotopic composition $\left(\delta^{13} \mathrm{C}\right)$ of the biphytanes derived from IPL-GDGT-0 and IPL-BDGT-0 together with the carbon isotopic composition $\left(\delta^{13} \mathrm{C}\right)$ of the total organic carbon (TOC), dissolved inorganic carbon (DIC) and methane.

\begin{tabular}{|c|c|c|c|c|c|c|c|}
\hline \# in dataset & core ID & sample depth (cmbsf) & $\delta^{13} \mathrm{C}(\mathrm{bp}-0$ IPL-GDGT) (\%) & $\delta^{13} \mathrm{C}(\mathrm{bp}-0$ IPL-BDGT) (\%о) & $\delta^{13} \mathrm{C}(\mathrm{TOC})(\%)$ & $\delta^{13} \mathrm{C}(\mathrm{DIC})(\% \circ)$ & $\delta^{13} \mathrm{C}(\mathrm{CH})(\% \circ)$ \\
\hline 5 & GeoB15103-1 & 459.5 & $-23 \pm 0.3$ & $-28.0 \pm 0.1$ & -20.1 & -7.9 & -67.8 \\
\hline 8 & GeoB15103-2 & 292.5 & $-22.5 \pm 0.03$ & $-28.7 \pm 0.1$ & -19.5 & -6.2 & -69.1 \\
\hline 20 & GeoB15105-2 & 112.5 & $-30.4 \pm 0.3$ & $-51.8 \pm 0.2$ & -24.8 & -19.9 & -88.5 \\
\hline 22 & GeoB15105-2 & 272.5 & $-24.3 \pm 0.2$ & $-41.0 \pm 0.8$ & -24.3 & -20.3 & -87.0 \\
\hline 34 & GeoB17306-1 & 32.5 & $-30.2 \pm 0.2$ & $-56.3 \pm 0.9$ & -26.9 & -4.1 & -63.6 \\
\hline 38 & GeoB17306-2 & 429.5 & $-24.7 \pm 0.3$ & $-53.0 \pm 0.2$ & -25.9 & 2.9 & -73.7 \\
\hline
\end{tabular}


Supplementary Figure S1. 1-D ${ }^{1} \mathrm{H}$ spectrum and expansions of BDGT-0 in $\mathrm{CDCl}_{3}$.

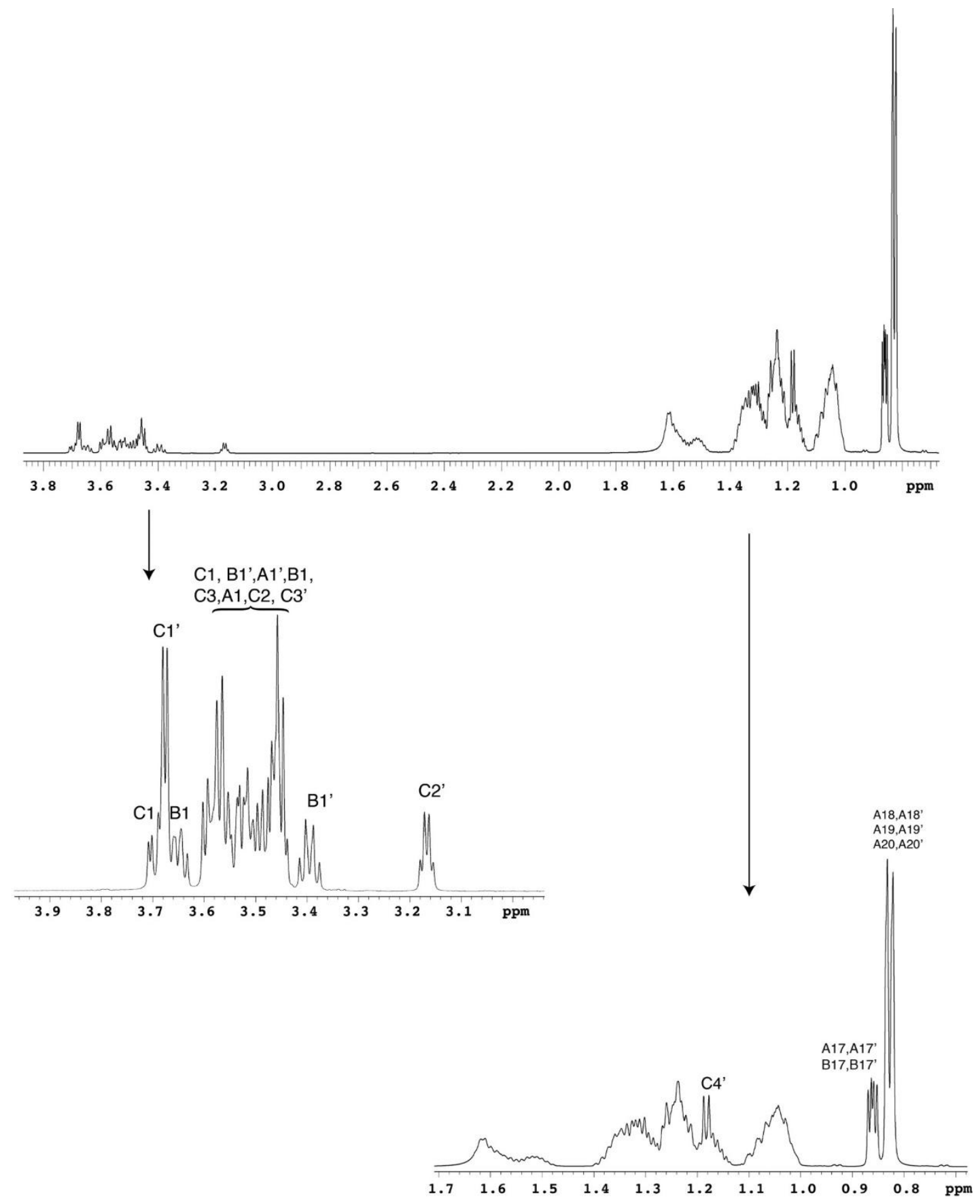


Supplementary Figure S2. ${ }^{1} \mathrm{H}-{ }^{13} \mathrm{C}$ HSQC 2-D spectra of BDGT-0.

Methine and Methyl signals are positive (black) and methylene signals are negative (red). A) Carbon atoms (A, A', B, B') 2 to (A, A', B, B') 20 and C4' (circled). C4' is well resolved in 2D from the remaining methyl signals. A3, A3', B3 and B3' although overlapped with each other are well resolved from the remaining branched alkyl chain methine groups $(7,11$ and 15$)$. B) Ether linked ${ }^{1} \mathrm{H}-{ }^{13} \mathrm{C}$ correlations and glycerol and butanetriol correlations. The butanetriol moiety gives a distinctive set of two well resolved methine signals (C2' and $\mathrm{C} 3$ ') that correlate with $\mathrm{C}^{\prime}$ ' in the ${ }^{1} \mathrm{H}^{13}{ }^{13} \mathrm{C} \mathrm{HMBC}$ experiment.

A
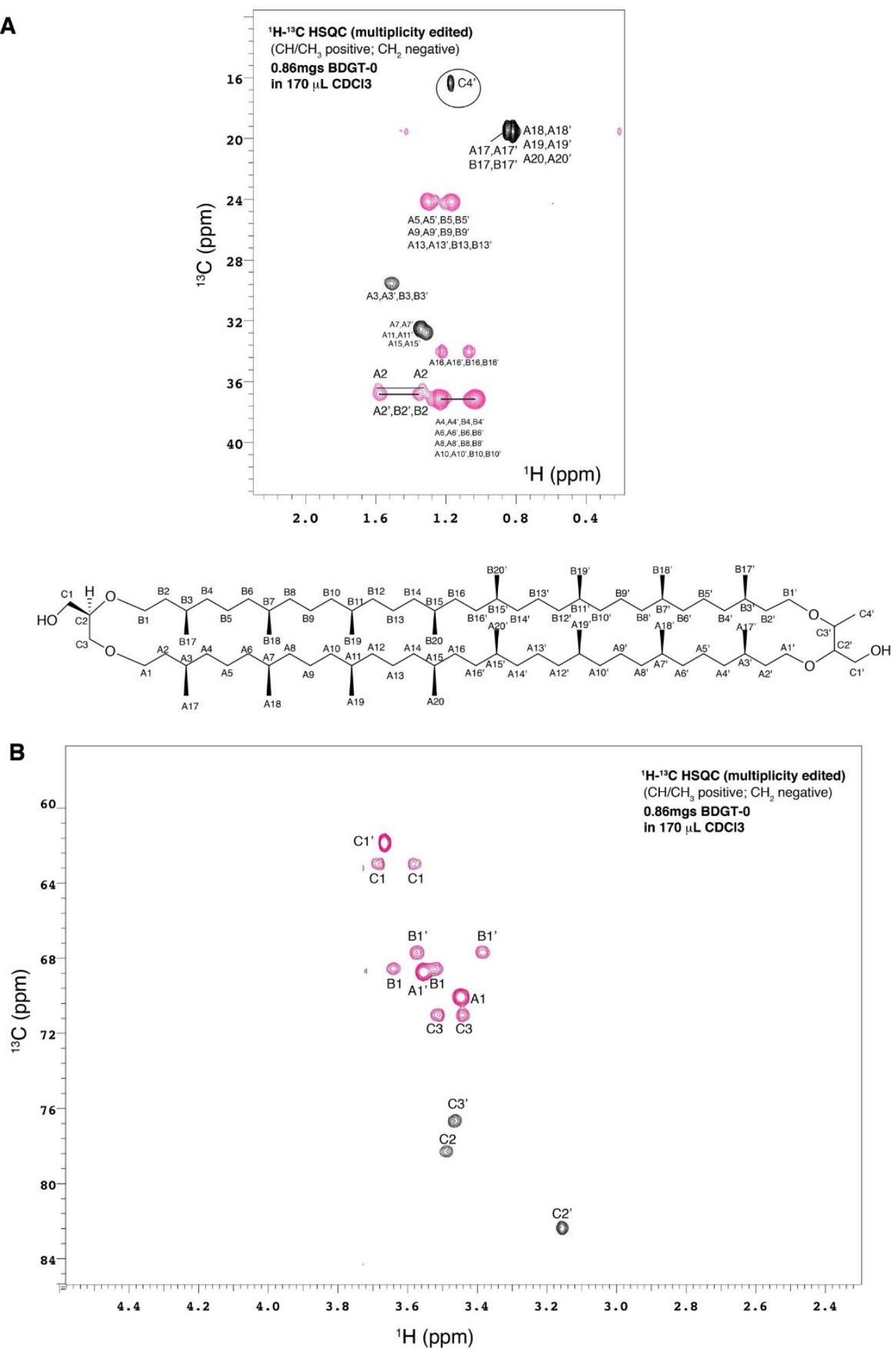
Supplementary Figure S3. ${ }^{1} \mathrm{H}-{ }^{13} \mathrm{C}$ HMBC 2-D spectra of BDGT-0.

A) Methyl and alkyl side-chain correlations. 17-methyls are resolved from 18, 19 and 20. A1 and A1' correlations allow discrimination of A2/A2' and A3/A3' chemical shifts. Weaker (due to multiplicity and non-degenerate protons) B1 and B1' correlations are visible but are just above the noise and not plotted at this level here. B) Carbon bonded to oxygen chemical shift region. Several key correlations are highlighted including key correlations from C4' to methine C3' and C2' (black box). Each of the correlation across the four ether linkages is highlighted.
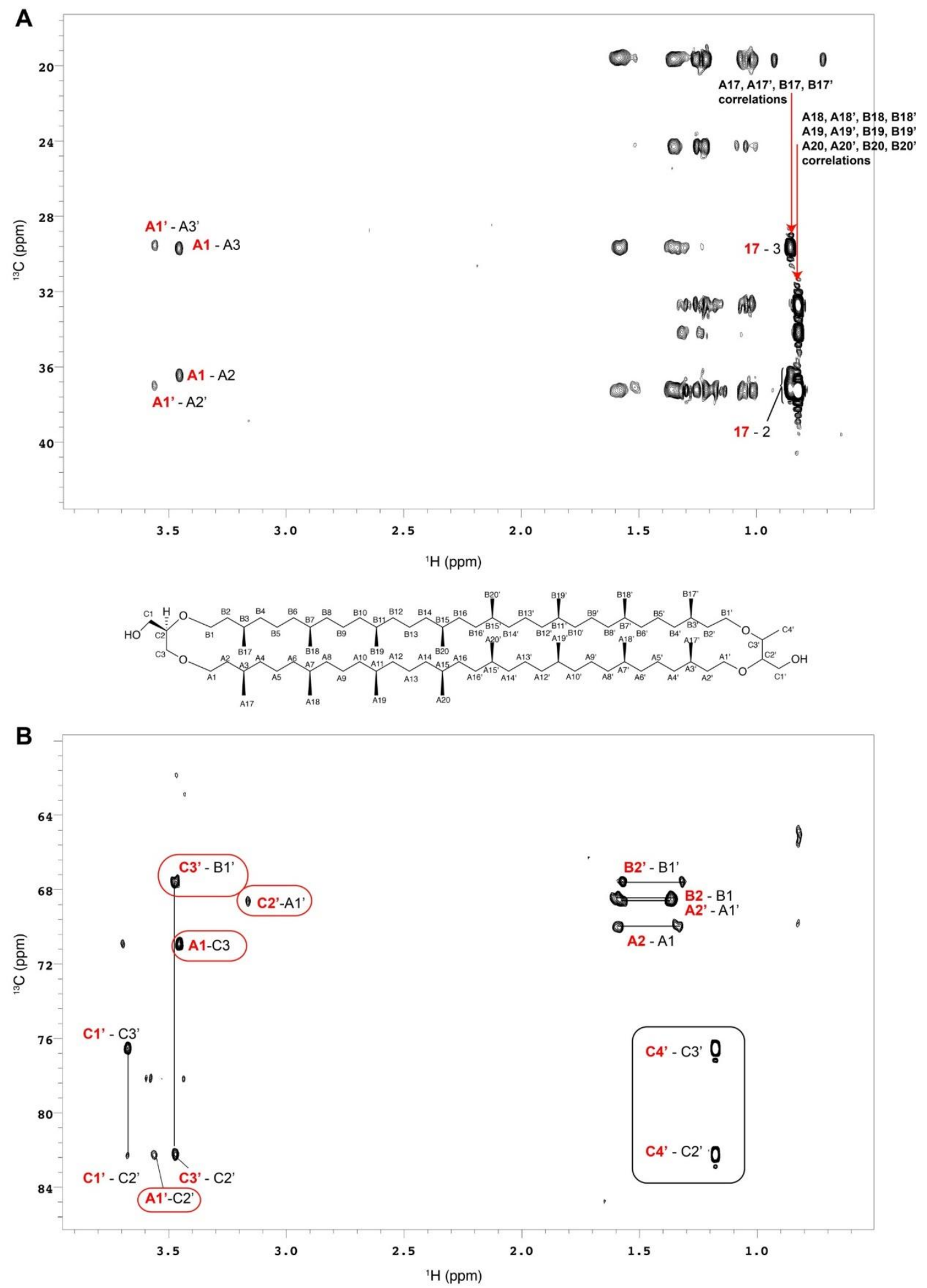